
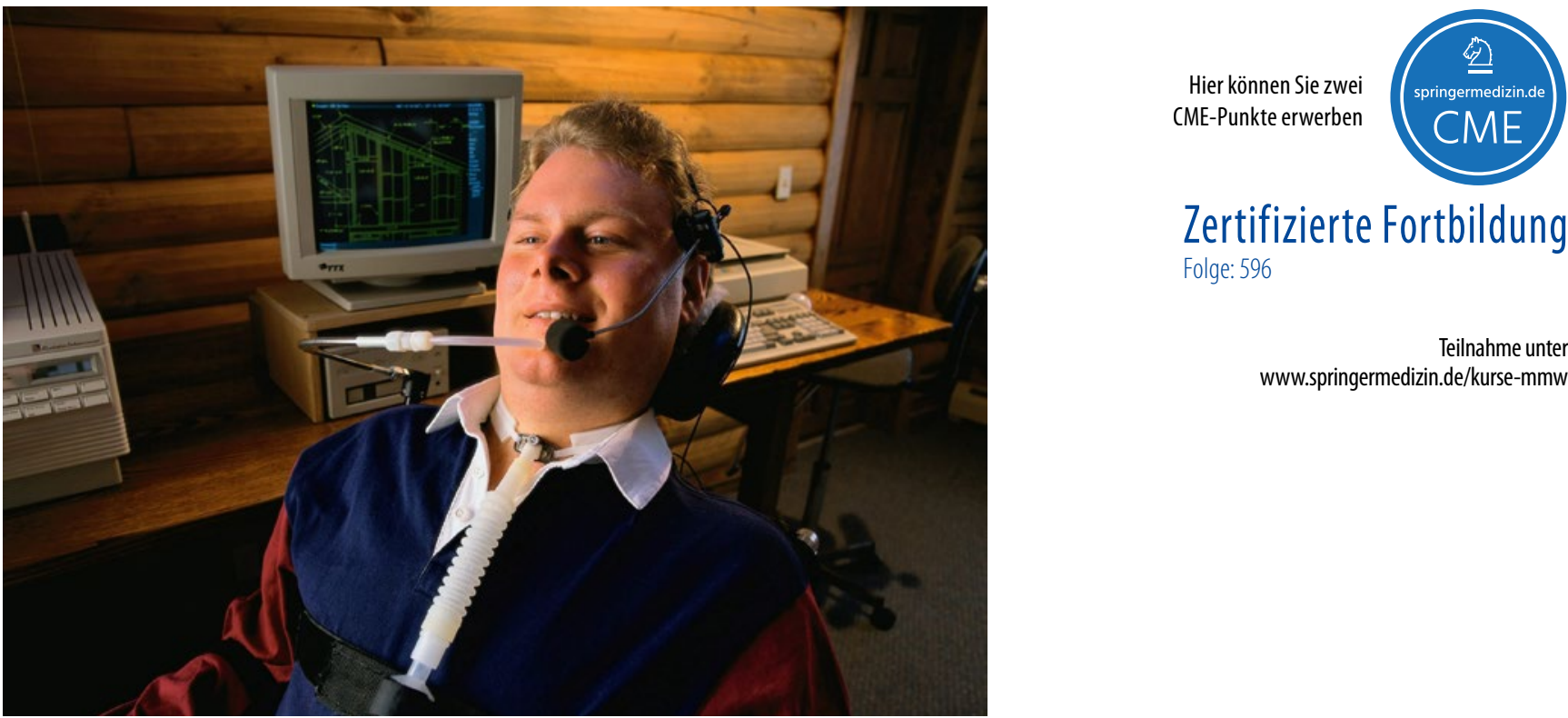

Teilnahme unter www.springermedizin.de/kurse-mmw

\title{
Außerklinische Beatmung
}

\author{
Lungenerkrankungen -- Autor: H. Schäfer
}

In den letzten Jahren ist eine deutliche und kontinuierliche Zunahme von meist schwerkranken Patienten zu konstatieren, die außerhalb einer Klinik beatmet werden. Diese Patienten stellen eine wachsende Herausforderung für alle an der Versorgung Beteiligten dar. In der Regel erfolgt die ärztliche Weiterbetreuung und die Verordnung der benötigten Hilfsmittel durch Hausärzte. In diesem Beitrag lesen Sie, was ein Hausarzt aus dem Bereich der Beatmungsmedizin wissen sollte.

Die für den zellulären Stoffwechsel des Menschen essenzielle Aufnahme von Sauerstoff $\left(\mathrm{O}_{2}\right)$ und Abgabe von Kohlendioxid $\left(\mathrm{CO}_{2}\right)$ erfolgt durch das respiratorische System. Dieses besteht aus der Lunge (gasaustauschendes System) und der Atempumpe (ventilierendes System), wobei beide Kompartimente unabhängig voneinander limitiert sein können (Abb. 1). Bei einer pulmonalen Störung ist nur eine Beeinträchtigung der Sauerstoffaufnahme erkennbar. Eine Beeinträchtigung der Atempumpfunktion geht dagegen sowohl mit einer Störung der Kohlendioxidabgabe als auch der Sauerstoffaufnahme einher. Grundsätzlich können Störungen beider Kompartimente sowohl akut als auch chronisch auftreten.
Eine akute ventilatorische Insuffizienz tritt meist bei einer akuten Verschlechterung einer vorbestehenden Beeinträchtigung auf, wie z. B. bei einer Exazerbation einer chronisch obstruktiven Lungenerkrankung (COPD). Kennzeichen ist die respiratorische Azidose.

Bei einer chronisch ventilatorischen Insuffizienz, z. B. bei neuromuskulären Erkrankungen, wird die respiratorische Azidose metabolisch durch eine Bikarbonatretention ausgeglichen. Letztlich können ganz unterschiedliche, prognostisch nicht einheitlich zu bewertende Krankheitsbilder zu einer chronisch ventilatorischen Insuffizienz führen (Tab. 1). Allen gemeinsam ist jedoch immer ein Missverhält-

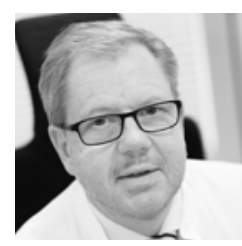

Prof. Dr. Harald Schäfer Innere Medizin-

Pneumologie, thorakale Onkologie, Palliativmedizin, Infektiologie, Schlaf-und Beatmungsmedizin, LungenZentrum Saar

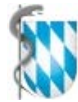

In Zusammenarbeit mit der Bayerischen Landesärztekammer 


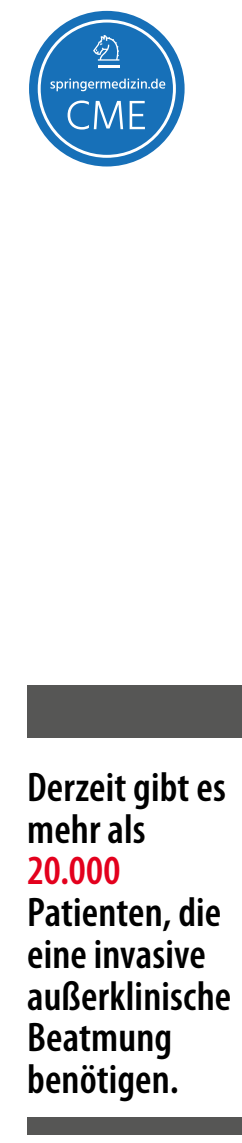
Tab. 1 Erkrankungen, die zu einer chronisch
ventilatorischen Insuffizienz führen

\begin{tabular}{l|l} 
Erkrankung & Erkrankungsbeispiele \\
\hline Neuromuskuläre & - Amyotrophe Lateralsklerose (ALS) \\
Erkrankungen & - Muskeldystrophien \\
& - spinale Muskelatrophie \\
& - Post-Polio-Syndrom \\
& - hohe Querschnittslähmung \\
& - Zwerchfellparese \\
\hline Thorakal-restriktive & - Kypho-)Skoliose \\
Erkrankungen & - M. Bechterew \\
& - Kielbrust, Trichterbrust \\
& - Post-TBC-Syndrom \\
& - restriktive Pleuraerkrankungen \\
& - posttraumatische oder postoperative \\
& Thoraxdeformität
\end{tabular}

Adipositas-(Obesitas)-Hypoventilationssyndrom

COPD/Lungenemphysem

nis zwischen Beanspruchung der Atempumpe und ihrer Kapazität (Abb. 2).

Therapeutisch kann eine respiratorische Insuffizienz leichteren Grades neben der Behandlung der Grunderkrankung mit einer Sauerstoffgabe ausgeglichen werden, bei chronisch respiratorischer Insuffizienz Typ I in Form einer Langzeitsauerstofftherapie. Eine manifeste ventilatorische Insuffizienz (Typ II) bedarf jedoch immer einer Beatmungstherapie.

Bei einer akuten ventilatorischen Insuffizienz erfolgt die Einleitung der Beatmung in der Regel unter intensivmedizinischen Bedingungen. Grundsätzlich ist die maschinelle Beatmung invasiv mittels Tubus bzw. Tracheostoma oder nicht-invasiv via Masken-

Abb. 1 Einteilung der respiratorischen Störungen nach der primären Störung der Kompartimente Lunge oder Atempumpe
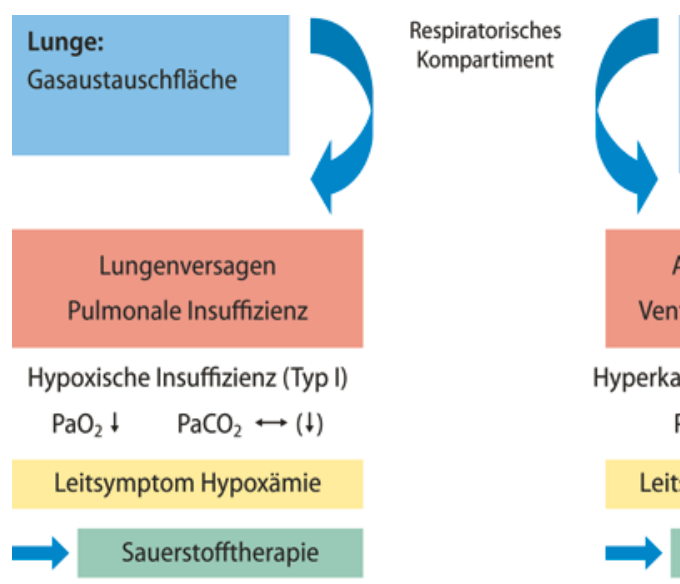

Atempumpe: Atemmuskulatur, Atemzentrum, Thorax, Nerven

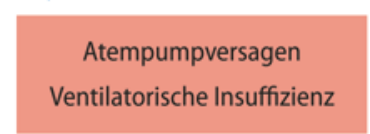

Hyperkapnische Insuffizienz (Typ II) $\mathrm{PaO}_{2} \downarrow \quad \mathrm{PaCO}_{2} \uparrow$

Leitsymptom Hyperkapnie

Beatmungstherapie

Pulmonale Insuffizienz = Oxygenierungsstörung; Ventilatorische Insuffizienz = Atempumpschwäche Respiratorische Störung bzw. Insuffizienz = übergeordneter Begriff

systemen (non-invasive Ventilation = NIV) möglich, wobei letzteres Verfahren primär zum Einsatz kommen sollte. Bei chronisch ventilatorischer Insuffizienz sollte nach Möglichkeit der nicht-invasiven Beatmung der Vorzug gegeben werden.

Eine solchermaßen im Krankenhaus fachkundig initiierte Maßnahme, die dann außerklinisch im häuslichen Umfeld weitergeführt wird, bietet in Form einer NIV nicht nur für den Patienten erhebliche Vorteile in Bezug auf Mobilität, Autonomie, Kommunikation, Schlucken und Sekretclearance, sondern verursacht auch im Vergleich zur invasiven Langzeitbeatmung erheblich geringere Kosten.

Eine invasive Langzeitbeatmung über ein Tracheostoma im außerklinischen Bereich ist nur bei ganz wenigen Indikationen erforderlich. Hierzu zählen im Wesentlichen fortgeschrittene neuromuskuläre Erkrankungen, bei denen eine kurzzeitige Unterbrechung der Beatmung bereits zu Luftnot oder einer lebensbedrohlichen Situation führt. Auch verbleibt letztlich ein Anteil von Patienten, bei denen es trotz aufwendiger Bemühung in einem spezialisierten Entwöhnungszentrum (= Weaningzentrum) nicht gelingt, eine dauerhafte Befreiung von der Beatmung zu erreichen.

\section{Epidemiologie und (Fehl-)Entwicklung der außerklinischen Beatmung}

In Deutschland ist die Zahl der Patienten mit invasiver außerklinischer Beatmung seit dem Jahr 2005 von etwa 1.000 Patienten insbesondere im letzten Jahrzehnt exponenziell auf schätzungsweise 20.000 Patienten gestiegen $[1,2,3)$. Die extreme $\mathrm{Zu}-$ nahme dieser Fallzahlen in den letzten zehn Jahren ist im Wesentlichen auf Patienten mit erfolgloser Beatmungsentwöhnung nach Akut-Intensivstationsaufenthalt zurückzuführen, und der überwiegende Teil dieser Patienten wird direkt von Akut-Intensivstationen nach Hause entlassen [4]. Inwieweit die aktuelle Corona-Pandemie zu einer weiteren Zunahme von langzeitbeatmeten Patienten führen wird, kann derzeit in keiner Weise abgeschätzt werden.

Nach einer aktuellen Erhebung ist auch die Zahl der Patienten mit einer Langzeitbeatmung (invasiv oder nicht-invasiv), die wiederum stationär behandelt werden, deutlich angestiegen [5]. Es ist also davon auszugehen, dass ein nicht unerheblicher Anteil der Patienten, die zu Hause langzeitbeatmet werden, aufgrund von Problemen oder Komplikationen, die im ambulanten Setting nicht behoben werden können, wieder einer stationären Behandlung bedürfen oder aber auch, dass keine entsprechenden ambulanten Versorgungsstrukturen vorhanden sind. Dabei handelt es sich vorwiegend um ältere ( $>70$ Jahre) $\mathrm{Pa}-$ tienten mit einer hohen Komorbidität und Einschränkungen mehrerer Organfunktionen, die so- 
wohl im ambulanten als auch im stationären Setting verschiedene Fachdisziplinen mit der entsprechenden Expertise erfordern [5].

Die Tatsache, dass auch Patienten mit hypoxischem Hirnschaden und demenziellem Syndrom langzeitbeatmet werden [5], langzeitbeatmete COPD-Patienten eine hohe Lebens-Unzufriedenheit haben und etwa die Hälfte der COPD-Patienten ihr Bedauern darüber zum Ausdruck bringt, dass sie im Rahmen des Intensivaufenthaltes tracheotomiert worden und nachfolgend langzeitbeatmet sind [6], wirft ethische Fragen auf. Nicht zuletzt belastet dies auch die finanziellen und personellen Ressourcen des deutschen Gesundheitssystems: Die häusliche Versorgung eines invasiv beatmeten Patienten bei einer 1:1 Betreuung durch Fachpflegepersonal kostet jährlich etwa 250.000.- $€$, die geschätzten Gesamtkosten belaufen sich jährlich auf etwa 2-4 Mrd. €. Eine qualitativ adäquate Versorgung ist angesichts des Mangels von entsprechend qualifiziertem Fachpersonal sowohl pflegerisch als auch ärztlich bei weitem nicht flächendeckend gesichert.

\section{Rechtliche Rahmenbedingungen und Strukturen}

Jeder Patient, auch derjenige mit der Notwendigkeit einer (invasiven) Beatmung, hat nach dem Sozialgesetzbuch (SGB) V das Recht auf eine häusliche Krankenpflege, wenn er seinem Aufenthaltsbestimmungsrecht folgend $\mathrm{zu}$ Hause versorgt werden möchte. Im Falle einer invasiven Beatmungstherapie ist dabei neben der Grundpflege eine 24-stündige Behandlungspflege notwendig, damit in einer Notsituation - z. B. durch Sekretverlegung der Trachealkanüle oder Störungen am Beatmungsgerät - jederzeit adäquat reagiert werden kann.

Die Fachgesellschaften haben Empfehlungen zur Qualifikation von Pflegekräften formuliert, die in Tab. 2 wiedergegeben sind [7]. Diese beziehen sich auf alle Einrichtungen, in denen außerklinische Beatmungspatienten betreut werden (Tab. 3). Auf Druck der Fachgesellschaften und der Kostenträger hin ist der Gesetzgeber zwischenzeitlich tätig geworden, um den Fehlentwicklungen und Fehlanreizen entgegenzutreten. Der vorgelegte Referentenentwurf eines Gesetzes zur Stärkung von Rehabilitation und intensivmedizinischer Versorgung (RISG) ist derzeit in der Diskussion.

\section{Team außerklinische Beatmung}

Neben dem Pflegeteam sind weitere Berufsgruppen an der Versorgung des Patienten im außerklinischen Bereich beteiligt [7]. Hierzu gehören Logopäden, Ergotherapeuten, Physiotherapeuten, Geräteprovider zur Ausstattung mit den verordneten Hilfsmitteln und deren technische Kontrolle sowie Ärzte
Tab. 2 Empfehlungen zur Qualifikation von Pflegediensten und Pflegefachkräften, die beatmete Patienten betreuen

- Pflegefachkräfte sollen Qualifizierungsmaßnahmen mit genau definiertem Inhalt und Umfang absolvieren - mindestens einen Basiskurs mit dem Abschluss „Pflegefachkraft für außerklinische Beatmung" (120-Stunden-Kurs).

- Pflegedienste, die beatmete Patienten betreuen, sollen für jeweils 12 Patienten mindestens eine Fachbereichsleitung mit den Zusatzqualifikationen „Atmungstherapeut" oder "Fachkrankenpflege für Anästhesie und Intensivmedizin" oder "Pflegeexperte für außerklinische Beatmung" vorhalten.

- Pflegehelfer, Arzthelfer oder Heilerziehungspflegende dürfen nicht eigenverantwortlich an der Betreuung beatmeter Patienten mitwirken. Vor der möglichen Übernahme von Grundpflegetätigkeiten innerhalb eines Pflegeteams sollten Personen dieser Berufsgruppen vom Pflegedienst eingearbeitet und spezifisch geschult werden.

Entsprechende Weiterbildungskurse (Pflegefachkraft für außerklinische Beatmung) werden u.a. von der Deutschen Interdisziplinären Gesellschaft für außerklinische Beatmung (DIGAB) organisiert.

(Allgemeinmediziner und Fachärzte). Der weiterbetreuende niedergelassene Arzt trägt die Verantwortung für die ambulante medizinische Behandlung eines Patienten mit außerklinischer Beatmung [7]. Um den Patienten fachlich beraten und Therapieziele formulieren zu können, sind Kenntnisse über den zu erwartenden Krankheitsverlauf, die Prognose und die pflegerischen und therapeutischen Maßnahmen erforderlich. Der Arzt soll daher Erfahrungen auf dem Gebiet der außerklinischen Beatmung aufweisen können, und er soll Hausbesuche durchführen [7].

Aufgrund der sektoralen Trennung und der in der Regel Nichtdurchführung von Haus- oder Heimbesuchen von Fachärzten liegt es auf der Hand, dass sich die meisten Allgemein- und Hausärzte, die im ambulanten Bereich für den Patienten zuständig sind, vielfach überfordert sehen. Im Idealfall sollten daher die formalen Rahmenbedingungen so gestaltet sein, dass für invasiv beatmete Patienten in der außerklinischen Versorgung ein Team außerklini-

Tab. 3 Versorgungs- bzw. Unterbringungskonzepte für beatmungspflichtige Patienten

\begin{tabular}{|l|l} 
Umfeld & $\begin{array}{l}\text { Charakterisierungsmerkmale mit Vor- und } \\
\text { Nachteilen }\end{array}$
\end{tabular}

\begin{tabular}{l|l} 
Häusliche Umgebung & -Gewohntes Umfeld
\end{tabular}

- Umgebung mit Angehörigen bzw. Bezugspersonen

- ggf. Umbaumaßnahmen erforderlich

-1:1-Betreuung mit Pflegefachkraft mit 24-stündiger Anwesenheit

Ambulant betreute Beatmungs-

- Option vermehrter Sozialkontakte durch Umgebung mit

Wohngemeinschaft

oder

stationäre Pflegeeinrichtung mit Spezialisierung auf außerklinische Beatmungspflege mehreren Patienten bzw. Personen mit ähnlicher Erkrankung

- fehlende Umgebung mit Angehörigen bzw. gewohnten Bezugspersonen

-1:3- bis 1:5-Betreuung durch Pflegefachkräfte 


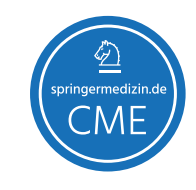

sche Beatmung bereit steht, bestehend aus Fachärzten (in der Regel Pneumologen oder Anästhesisten mit entsprechender Erfahrung), Atmungstherapeuten und Mitarbeiter eines Beatmungs-Pflegedienstes, die sich um die sehr speziellen Belange dieser Patienten kümmern und ungeplante Klinikeinweisungen nach Möglichkeit verhindern. Darüber hinaus sollte die Zusammenarbeit mit einem Beatmungszentrum bestehen (Abb. 3).

\section{Beatmungszentrum}

Entsprechend den Empfehlungen der aktuellen Deutschen Leitlinie [7] sollte ein Beatmungszentrum neben entsprechender Expertise mit einer

Abb. 2 Erkrankungen mit Störungen der Atemmechanik, die zu einer chronisch ventilatorischen Insuffizienz führen können aufgrund chronischer Überbelastung oder verminderter (Atem-)Muskelkraft

\section{Chronische Über-/Fehlbelastung \\ Muskelschwäche}

\begin{tabular}{c|c}
\hline$\cdot$ COPD/Emphysem & $\cdot$ Muskedystrophie \\
$\cdot$ (Kypho-)Skoliose & $\cdot$ Poliomyelitis \\
$\cdot$ Post-TBC-Syndrom & $\cdot$ Amyotrophe Lateralsklerose \\
$\cdot$ Mukoviszidose & $\cdot$ Myasthenia gravis \\
$\cdot$ Adipositas & $\begin{array}{l}\text {-Guillain-Barré-Syndrom } \\
\cdot \text { Zwerchfellparese }\end{array}$ \\
\hline $\begin{array}{c}\text { Aktuelle Beanspruchung } \\
\text { (Last) }\end{array}$ & $\begin{array}{l}\text { Maximalkraft } \\
\text { (Kapazität) }\end{array}$ \\
\hline
\end{tabular}

$A b b .3$ Organisation der Versorgung von Beatmungspatienten im stationären und außerklinischen Bereich und der beteiligten Berufsgruppen im außerklinischen Bereich
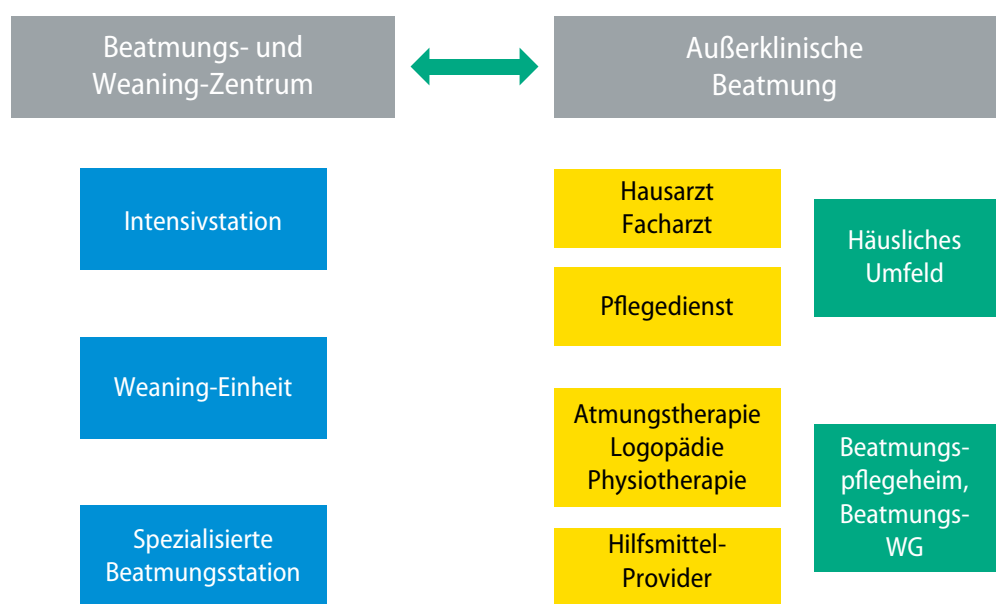

Beatmungsstation
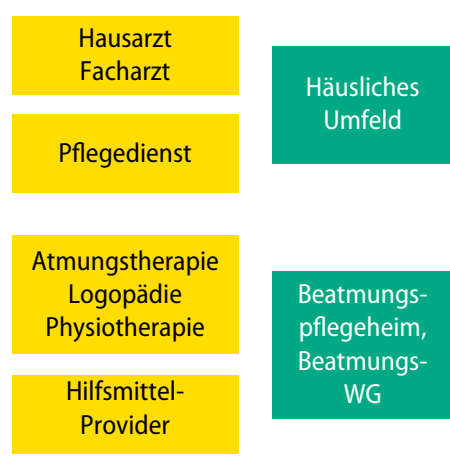

BeatmungsBeatmungsWG pflegeheim,
Intensivstation auch eine spezialisierte Normalstation für invasiv und nicht-invasiv beatmete $\mathrm{Pa}$ tienten vorhalten und über eine Weaning-Einheit verfügen. Eine solche Strukturierung ist notwendig, da bei einem Großteil der Patienten die invasive außerklinische Beatmung infolge einer akuten Verschlechterung der Grunderkrankung bzw. einem längeren Intensivaufenthalt und Multimorbidität erfolgt. Nur bei ca. 38\% der in ein pneumologisches Weaningzentrum verlegten Patienten mit vermeintlichem Weaningversagen liegt dieses tatsächlich auch vor [8]. Mitunter kann auch im Verlauf nach primärem Weaningversagen und Überführung in die außerklinische Beatmung durchaus noch Weaningpotenzial bestehen, sodass die Fortführung einer intensiven physiologischen und logopädischen Therapie außerklinisch indiziert sein kann und dann in einem zweiten Schritt erneut eine Überprüfung und ggf. die Durchführung einer Beatmungsentwöhnung in einem entsprechenden Zentrum sinnvoll sein können [7,9]. Daher sollte der betreuende Hausarzt mit dieser Fragestellung Kontakt mit einem Beatmungs- und Entwöhnungs-Zentrum aufnehmen, falls der Patient aus einer anderweitigen Einrichtung entlassen wurde.

Generell sollte eine invasive außerklinische Beatmung möglichst vermieden werden. Auch ist grundsätzlich immer zu prüfen, ob die invasive Beatmung in eine nicht-invasive Beatmung überführt werden kann [7]. Da die Entwöhnung vom Respirator und insbesondere auch die Entscheidung und Durchführung einer Dekanülierungsmaßnahme stets ein komplexes Behandlungskonzept erfordert, ist dies nur im Rahmen eines stationären Settings und nicht ambulant möglich $[7,9]$.

\section{Überleitung in die außerklinische Beatmung, intersektorale Schnittstellenversorgung}

Die Entlassung eines Patienten aus der Klinik in die außerklinische Beatmung darf nur in stabilem Zustand erfolgen und bedeutet aufgrund des notwendigen Entlass- und Überleitmanagements einen nicht unerheblichen logistischen Aufwand mit einem entsprechenden zeitlichen Vorlauf. Hierzu bedarf es einer speziellen Expertise. Insbesondere sind vorab vom Beatmungszentrum zu organisieren bzw. festzulegen: notwendige Geräte und Hilfsmittel, Beatmungseinstellungen, weitere Maßnahmen wie evtl. zusätzliche Sauerstoffzufuhr, Sekretmanagement, Logopädie, Physiotherapie, Ernährungsmanagement sowie die Sicherstellung der pflegerischen Versorgung und der speziellen Beatmungspflege in dem entsprechenden Umfeld, die Kostenübernahme der Hilfsmittel und die pflegerischen Versorgung.

Erforderlich sind eine schriftliche Fixierung und Information des weiterbetreuenden Arztes und 
Hausarztes. Auch sollte eine Festlegung bzgl. weiterer Kontrolluntersuchungen erfolgen.

\section{Ethische Aspekte}

Ethische Überlegungen und Grundsätze spielen im Rahmen der Intensiv- und Beatmungsmedizin eine wesentliche Rolle, wenn es darum geht, Therapieziele festzulegen und ggf. auch Therapiezieländerungen und Therapiebegrenzungen oder -abbrüche zu formulieren. Letztlich geht es darum, Langzeitbeatmungen zu vermeiden (fehlende Indikation, Ablehnung durch den Patienten).

Ethische Aspekte müssen auch bei Entscheidungsfindungen bei allen fortgeschrittenen chronisch respiratorischen Erkankungen, insbesondere bei Komorbidität bzw. am Lebensende berücksichtigt werden. Im Sinn einer vorausschauenden Therapieplanung sollten in Beratungs- und Informationsgesprächen gemeinsam mit dem Patienten und seinen Bezugspersonen mögliche Szenarien erörtert und Maßnahmen festgelegt werden, nach Möglichkeit in schriftlicher Form (z. B. Patientenverfügung). Es ist eine zentrale Aufgabe des behandelnden Hausarztes in Abstimmung mit dem Facharzt bzw. auch einem entsprechenden Zentrum mit dem Patienten aktiv das Gespräch zu suchen. Dieser Aspekt gewinnt aktuell unter den Bedingungen des zunehmenden Patientenaufkommens in der Intensivmedizin aufgrund der COVID-19-Erkrankungen nochmals eine besondere Bedeutung.

Auch bei Patienten mit etablierter außerklinischer Maskenbeatmung (NIV) sind entsprechende Überlegungen und Maßnahmen unabdingbar. So kann bei fortschreitender Grunderkrankung bei sich dann abzeichnender dauerhafter Beatmungsabhängigkeit (z.B. bei fortschreitender COPD oder amyotropher Lateralsklerose) die Entscheidung zum Übergang in eine invasive Beatmung mit allen Konsequenzen anstehen oder aber eine Therapiebegrenzung und ein palliatives Konzept im Vordergrund stehen.

\section{Nicht-invasive außerklinische Beatmung}

Eine NIV bietet für Patienten nicht nur erhebliche Vorteile in Bezug auf Mobilität, Autonomie, Kommunikation, Schlucken und Sekretclearance, sondern verursacht auch erheblich geringere Kosten im Vergleich zur invasiven Langzeitbeatmung. Die Einleitung einer NIV muss immer unter stationären Bedingungen erfolgen, da dies einer mehrtägigen (und nächtlichen) Einübungs- und Eingewöhnungsphase unter fachkundiger Anleitung und Vorhaltung bestimmter apparativer Untersuchungs- und Kontrollverfahren bedarf. Darüber hinaus ist eine regelmäßige Betreuung notwendig.

Es gibt eine Vielzahl von Maskensystemen und Heimrespiratoren, die jeweils individuell zum Ein- satz kommen. Der Patient sollte zu einer selbständigen Maskenanlage sowie Ein- und Ausschalten des Beatmungsgerätes in der Lage sein, im Einzelfall kann dies auch durch eine Betreuungsperson erfolgen. Eine ausführliche Instruktion und Einübung ist unabdingbar für die langfristige Therapieadhärenz. In der Regel erfolgt eine intermittierende Selbstbeatmung über 6-8 Stunden, vorwiegend nachts, es können jedoch auch längere Beatmungszeiten notwendig sein. Im Folgenden werden die wesentlichen Indikationen und Besonderheiten dargestellt.

\section{Thorakal-restriktive Erkrankungen}

Eine chronisch ventilatorische Insuffizienz bei thorakal-restriktiven Erkrankungen ist seit Jahren eine etablierte Indikation zur Durchführung einer NIV [7]. Wesentliches Kriterium zur Einleitung ist der Nachweis einer chronischen Hyperkapnie am Tag mit typischen Symptomen der ventilatorischen Insuffizienz und Lebensqualitätseinschränkung wie verminderter körperlicher Belastbarkeit, Tagesmüdigkeit und Konzentrationsstörungen, Schlafstörungen bzw. nächtliche Dyspnoe. Indiziert ist eine NIV auch bei

- chronischer Tageshyperkapnie mit $\mathrm{PaCO}_{2} \geq 45$ $\mathrm{mmHg}$

- nächtlicher Hyperkapnie mit $\mathrm{PaCO}_{2} \geq 50 \mathrm{mmHg}$ oder bei

-Normokapnie am Tag mit Anstieg des transkutanen $\mathrm{pCO}_{2} \mathrm{um} \geq 10 \mathrm{mmHg}$ in der Nacht.

Bei Patienten ohne manifeste Hyperkapnie aber schwerer restriktiver Ventilationsstörung (Vitalkapazität $<50 \%$ des Sollwertes) sollen kurzfristige klinische Kontrolluntersuchungen innerhalb von drei Monaten erfolgen, einschließlich einer nächtlichem Polygrafie. Hierbei lassen sich dann auch ggf. längerstreckige Sauerstoffentsättigungen als Korrelat einer nächtlichen Hypoventilation nachweisen.

Die NIV in Form einer druck- oder volumengesteuerten Beatmung führt in dieser Indikation durch die Entlastung der Atemmuskulatur und Verbesserung des Gasaustausches meist zu einer vollständigen Beseitigung der Symptomatik mit einer erheblichen Verbesserung der Leistungsfähigkeit und Lebensqualität [7] sowie der Aufrechterhaltung der Berufstätigkeit. Da die Grunderkrankung meist stabil ist, kann die Therapie über Jahre bzw. Jahrzehnte durchgeführt werden. In Beobachtungsstudien wurde bei dieser Patientengruppe ein verbessertes Langzeitüberleben durch die NIV nachgewiesen [7].

\section{Neuromuskuläre Erkrankungen (NME)}

Diese Erkrankungsgruppe ist sehr heterogen. Aufgrund der in der Regel betroffenen peripheren Muskulatur ist eine klinische Belastungsdyspnoe kein valides Kriterium für eine Beteiligung der Atem-

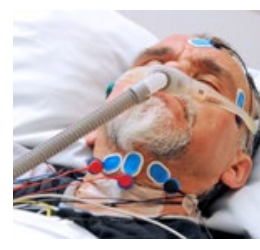

Mittels einer Polysomnografie lassen sich Atempumpenstörungen nachweisen. 
pumpmuskulatur, sodass weitere Untersuchungen wie eine Lungenfunktion und eine Messung der Atemmuskelkraft sowie der Blutgase notwendig sind. Solche Untersuchungen sollten bei NME in regelmäßigen Abständen erfolgen. Bei einer Verminderung der forcierten Vitalkapazität $<70 \%$ des Sollwerts wird die Durchführung einer Poly(somno)grafie, ggf. auch eine transkutane $\mathrm{CO}_{2}$-Messung empfohlen, da sich hierbei dann unter Umständen als Frühzeichen einer latenten Atempumpinsuffizienz nächtliche Hypoventilationen, zunächst im REM-Schlaf, detektieren lassen (Abb. 4). Nicht selten lassen sich dann aufgrund einer Mitbeteiligung der pharyngealen Muskulatur auch obstruktive Apnoen erkennen.

Eine gewisse Sonderform nimmt die amyotrophe Lateralsklerose ein, da hierbei mitunter rasch progrediente Verläufe zu beobachten sind und die Prognose generell ohne Beatmung ungünstig ist. In diesen Fällen kann eine NIV bei einer forcierten Vitalkapazität (FVC) $<70 \%$ und einer Abnahme der FVC um mehr als 10\% innerhalb von drei Mo- naten aufgrund des raschen Erkrankungsverlaufs unabhängig von weiteren Untersuchungsbefunden begonnen werden [7]. Auch stellt diese Patientengruppe eine besondere Herausforderung im Management dar, da häufig eine Mitbeteiligung der pharyngealen Muskulatur (bulbäre Symptomatik) und der Handmuskulatur gegeben ist. Mit diesen Patienten muss daher frühzeitig über eine (elektive) Tracheotomie und invasive Langzeitbeatmung gesprochen werden.

$\mathrm{Zu}$ beachten ist bei Patienten mit NME auch die in der Regel eingeschränkte Hustenkapazität und Sekretclearance, sodass eine diesbezügliche Prüfung und ggf. eine spezielle Therapie in Form von assistiven Hustentechniken notwendig sind. Insofern ist bei Patienten mit NME die Wahl des Beatmungsverfahrens und Zugangs (NIV oder invasiv) neben dem individuellen Patientenwunsch in besonderem Maße abhängig von

- dem Ausmaß der Muskelschwäche im oropharyngealen Bereich und Mundschluss,

Abb. 4 Polysomnogramm einer Patientin mit Muskeldystrophie und Nachweis von ausschließlich REM-Schlafassoziierten Sauerstoffentsättigungen infolge Hypoventilationen. Hier kommt es aufgrund der im REM-Schlaf deutlich veränderten muskulären Kontrolle, die auch die Atemmuskulatur betrifft, zu einer abgeflachten Atmung (Hypoventilation) mit ausgeprägter Sauerstoffentsättigung und Herzfrequenzanstieg. Aufgrund der begleitenden nächtlichen Hyperkapnie berichten solche Patienten meist über morgendliche Kopfschmerzen und Unausgeschlafenheit.
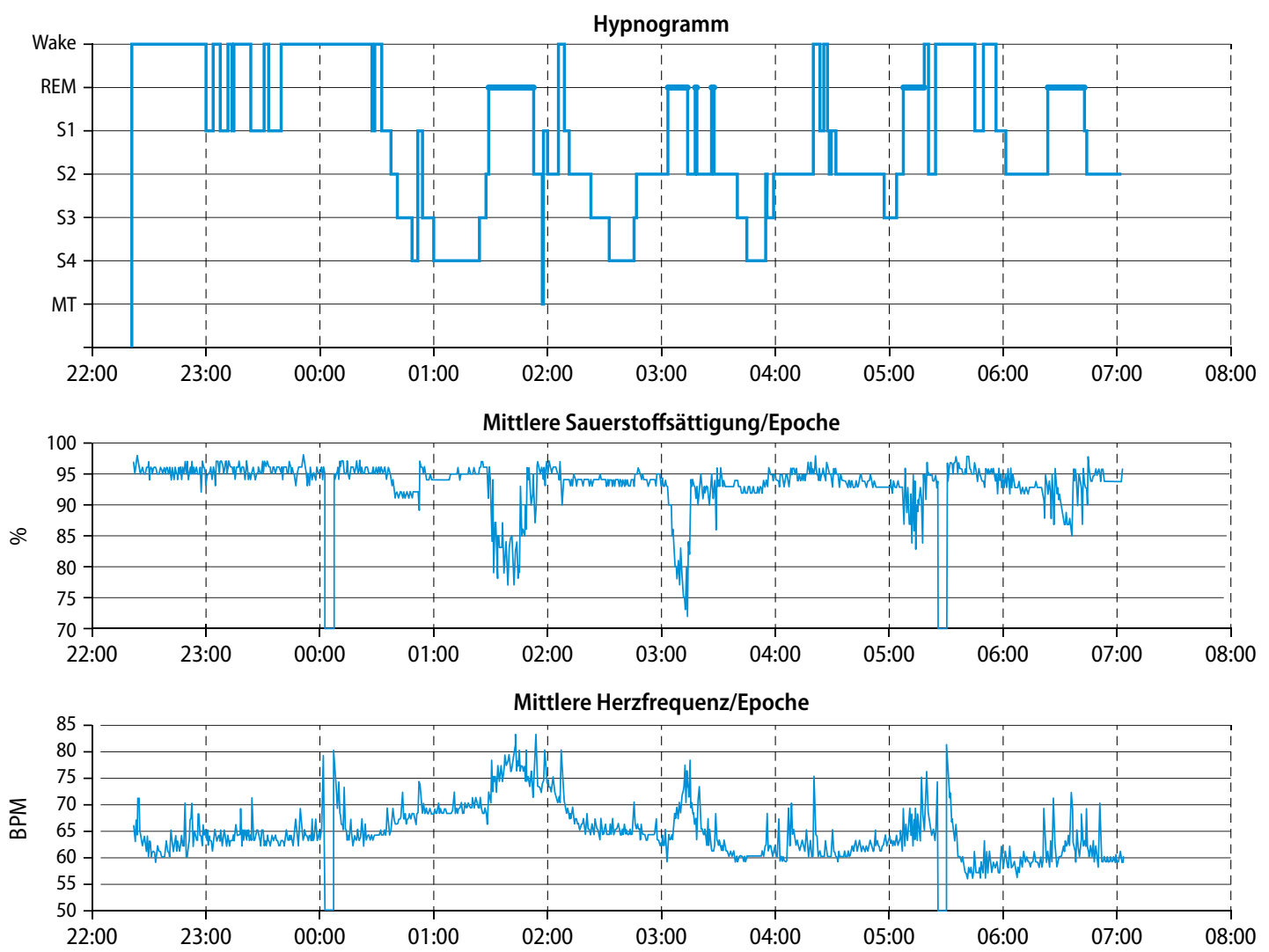
- den bulbären Symptomen mit dem Risiko rezidivierender Aspirationen und

- einer Hustenschwäche mit unzureichender Sekretclearance, die mit assistiven Hustentechniken nicht zu kompensieren ist.

\section{Obesitas-Hypoventilations-Syndrom}

Dieses Syndrom ist definiert als eine Adipositas (Body-Mass-Index $[\mathrm{BMI}] \geq 30 \mathrm{~kg} / \mathrm{m}^{2}$ ) in Kombination mit einer chronisch alveolären Hypoventilation und konsekutiver Tageshyperkapnie $\left(\mathrm{PaCO}_{2} \geq\right.$ $45 \mathrm{mmHg}$ ) unter Ruheatmung nach Ausschluss anderer bekannter Ursachen einer Hypoventilation. Da eine hohe Koinzidenz bzw. Überlappung mit der obstruktiven Schlafapnoe besteht, ist eine poly(somno)grafische Untersuchung obligat.

Unbehandelte Patienten haben neben der typischen Symptomatik auch ein deutlich erhöhtes Mortalitätsrisiko, weshalb eine Continuous-positive-airwaypressure(CPAP)-Beatmung oder eine NIV indiziert sind. Hier sind im Verlauf regelmäßige poly(somno) grafische sowie Blutgas-Untersuchungen notwendig. Mitunter kann nach drastischer Gewichtsreduktion ein NIV-Auslassversuch erfolgen.

\section{Chronisch obstruktive Atemwegserkrankung (COPD)}

Nachdem die außerklinische NIV bei symptomatischen Patienten mit stabiler COPD und Tageshyperkapnie $\left(\mathrm{PaCO}_{2} \geq 50 \mathrm{mmHg}\right)$ die Blutgassituation, die klinische Symptomatik und die körperliche Belastungsfähigkeit Betroffener verbessert [7], wurde nun in einer prospektiven randomisierten kontrollierten Studie erstmals auch eine Prognoseverbesserung durch die NIV nachgewiesen [10]. Hier zeigte sich eine Reduktion der Einjahresmortalität von 33\% auf $12 \%$, wenn es gelang, durch die NIV tagsüber eine Normalisierung der Hyperkapnie zu erreichen [10]. Hierfür ist jedoch in der Regel eine hohe Beatmungsintensität mit hohen inspiratorischen Beatmungsdrücken (meist zwischen 22 und 30 mbar), eine sog. „high-intensity-NIV“ erforderlich.

Angemerkt sei noch, dass die Einleitung einer NIV bei fortgeschrittener COPD und nach oben genannten Kriterien ein hohes Maß an Motivation und Mitarbeit des Patienten erfordert und ein entsprechendes Engagement des therapeutischen Teams. Deshalb kann die stationäre Behandlungszeit bis zum Erreichen einer stabilen Therapieeinstellung als Voraussetzung für eine langfristige Therapieadhärenz bei den meist multimorbiden Patienten durchaus bis zu zwei Wochen in Anspruch nehmen.

Etabliert ist die NIV als Akutmaßnahme bei Exazerbationen einer COPD mit akuter ventilatorischer Insuffizienz sowohl in symptomatischer als auch prognostischer Hinsicht durch die Vermeidung einer in- vasiven Beatmung. Oft wird bei diesen Patienten die in der Akutphase eingeleitete NIV als Heimbeatmungstherapie fortgeführt, wobei der Stellenwert eines solchen Vorgehens bisher nicht klar war.

Allerdings konnte kürzlich in einer randomisiert kontrollierten Studie gezeigt werden, dass Patienten, die nach stationärer Behandlung aufgrund einer Akutexazerbation eine über zwei Wochen persistierende Hyperkapnie aufweisen, durch die NIV einen prognostischen Benefit hinsichtlich der stationären Wiederaufnahme oder des Versterbens innerhalb von 12 Monaten haben [11]. Daher sollte bei solchen Patienten zwei Wochen nach Krankenhausentlassung nochmals eine Blutgasanalyse erfolgen und bei persistierender Hyperkapnie am Tag $\left(\mathrm{PaCO}_{2} \geq\right.$ $53 \mathrm{mmHg}$ ) die Indikation zu einer NIV-Einleitung geprüft werden.

\section{Literatur: springermedizin.de/mmw}

Title:

Home mechanical ventilation

Keywords:

Home mechanical ventilation, long term vantilation, NIV, tracheostomy

\section{Anschrift des Verfassers:}

\section{Prof. Dr. med. Harald Schäfer}

Lungenzentrum Saar

SHG Kliniken Völklingen

Richardstrasse 5-9

D-66333 Völklingen

E-Mail:h.schaefer@vk.shg-kliniken.de

\section{FAZIT FÜR DIE PRAXIS}

1. Die außerklinische Beatmung ist in Bezug auf Indikation, Methodik und Outcome-Parameter wissenschaftlich etabliert.

2. Eine flächendeckende konzeptionelle und geregelte interdisziplinäre und vor allem intersektorale Versorgung der Patienten inklusive differenzierter Abrechnungsmodalitäten ist in Deutschland bis dato nicht existent.

3. Dem Hausarzt kommt in diesem Spannungsfeld nicht nur als Behandler, sondern auch als Berater, Vermittler und Weichensteller eine besondere Rolle zu.

4. Bei invasiv außerklinisch beatmeten Patienten sollte von Seiten des Hausarztes immer ein enger Informationsaustausch mit den übrigen an der Behandlung beteiligten Personen und dem Beatmungszentrum gesucht werden.

\section{INTERESSENKONFLIKT}

Der Autor erklärt, dass er sich bei der Erstellung des Beitrags von keinen wirtschaftlichen Interessen leiten ließ. Er legt folgende potenzielle Interessenkonflikte offen: keine.

Der Verlag erklärt, dass die inhaltliche Qualität des Beitrags von zwei unabhängigen Gutachtern geprüft wurde. Werbung in dieser Zeitschriftenausgabe hat keinen Bezug zur CME-Fortbildung. Der Verlag garantiert, dass die CME-Fortbildung sowie die CME-Fragen frei sind von werblichen Aussagen und keinerlei Produktempfehlungen enthalten. Dies gilt insbesondere für Präparate, die zur Therapie des dargestellten Krankheitsbildes geeignet sind. 


\section{Außerklinische Beatmung}

Teilnehmen und Punkte sammeln können Sie

- als e.Med-Abonnent von SpringerMedizin.de

- als registrierter Abonnent dieser Fachzeitschrift

- zeitlich begrenzt unter Verwendung der abgedruckten FIN.
Dieser CME-Kurs ist zwölf Monate auf SpringerMedizin.de/CME verfügbar. Sie finden ihn, wenn Sie die FIN oder den Titel des Beitrags in das Suchfeld eingeben. Alternativ können Sie auch mit der Option „Kurse nach Zeitschriften" zum Ziel navigieren.
? Welche Aussage trifft zu? Kennzeichen einer ventilatorischen Insuffizienz ist

$\bigcirc$ eine Alkalose in der Blutgasanalyse.

$\bigcirc$ eine verminderte Sauerstoffsättigung.

$\bigcirc$ eine erhöhte Atemfrequenz.

$\bigcirc$ eine Hyperkapnie in der Blutgasanalyse.

$\bigcirc$ eine Dyspnoesymptomatik unter Belastung.

? Welche Aussage trifft zu? Bei einer chronisch ventilatorischen Insuffizienz

$\bigcirc$ ist blutgasanalytisch meist aufgrund der kompensatorischen renalen Bikarbonatretention keine Azidose nachweisbar,

$\bigcirc$ ist die Sauerstoffgabe die Therapie der Wahl,

$\bigcirc$ lässt sich die Ursache immer lungenfunktionsanalytisch ermitteln.

$\bigcirc$ brauchen neuromuskuläre Ursachen nicht in Betracht gezogen werden.

$\bigcirc$ haben Patienten keine Belastungsdyspnoe.

? Welche Aussage trifft nicht zu? Bei folgenden Erkrankungen, insbesondere im fortgeschrittenen Stadium, ist mit der Entwicklung einer chro- nisch ventilatorischen Insuffizienz zu rechnen:

thorakal-restriktive Erkrankungen.

neuromuskuläre Erkrankungen.

Asthma bronchiale.

$\bigcirc$ COPD/Lungenemphysem.

schwere Adipositas.

? Welche Aussage zur nicht-invasiven Heimbeatmungstherapie (NIV) trifft zu?

$\bigcirc$ Die Einleitung erfolgt in der häuslichen Umgebung unter fachärztlicher Aufsicht.

$\bigcirc$ Die Einleitung erfolgt unter stationären Bedingungen.

Eine Kontrolluntersuchung nach erfolgreicher Einleitung erübrigt sich.

$\bigcirc$ Die Therapie muss bei einem ObesitasHypoventilationssyndrom lebenslang erfolgen.

$\bigcirc$ Die Indikation bei COPD- Patienten ist rein symptomatisch. Prognostische Überlegungen sind zu vernachlässigen.

? Welche Aussage zur nicht-invasiven Heimbeatmungstherapie (NIV) trifft nicht zu?

Wesentliches Kriterium zur Einleitung ist eine klinische Symptomatik sowie der Nachweis einer Tageshyperkapnie.

$\bigcirc$ Die Durchführung einer Poly(somno)- grafie kann wesentliche Zusatzinformationen ergeben.

$\bigcirc$ Bei Patienten mit thorakal-restriktiven Erkrankungen führt eine erfolgreiche NIV meist zu einer drastischen Lebensqualitätsverbesserung und Symptomfreiheit.

Patienten mit amyotropher Lateralsklerose (ALS) sollten bereits bei ersten Symptomen einer invasiven Langzeitbeatmung zugeführt werden.

P Patienten mit fortgeschrittenen neuromuskulären Erkrankungen haben oftmals eine eingeschränkte Hustenkapazität.

? Wer gehört nicht zum Team außerklinische Beatmung?

$\bigcirc$ Beatmungspflegeteam.

$\bigcirc$ Logopädie.

$\bigcirc$ Atmungstherapie.

Physiotherapie.

Krankenkassenmitarbeiter.

? Welche Aussage zur invasiven Beatmung trifft zu?

$\bigcirc$ Die Respiratorentwöhnung in einem spezialisierten Weaning-Zentrum gelingt immer, wenn man sich genug Zeit nimmt.
Dieser CME-Kurs wurde von der Bayerischen Landesärztekammer mit 2 Punkten in der Kategorie I zur zertifizierten Fortbildung freigegeben und ist damit auch für andere Ärztekammern anerkennungsfähig.
Für eine erfolgreiche Teilnahme müssen 70\% der Fragen richtig beantwortet werden. Pro Frage ist jeweils nur eine Antwortmöglichkeit zutreffend. Bitte beachten Sie, dass Fragen wie auch Antwortoptionen online abweichend vom Heft in zufälliger Reihenfolge ausgespielt werden.
Bei inhaltlichen Fragen erhalten Sie beim Kurs auf SpringerMedizin.de/CME tutorielle Unterstützung. Bei technischen Problemen erreichen Sie unseren Kundenservice kostenfrei unter der Nummer (0800) 7780777 oder per Mail unter kundenservice@springermedizin.de. 
Stabile Patienten sollten zur Respiratorentwöhnung in stationäre Pflegeeinrichtungen geschickt werden.

$\bigcirc$ Die Verordnung einer Beatmungspflege bei invasiv heimbeatmeten Patienten sollte aus Kostengründen unterbleiben.

Invasiv heimbeatmete Patienten sollten mit der Frage einer Respiratorentwöhnung in einem Weaning-Zentrum vorgestellt werden.

$\bigcirc$ Die potenzielle Umstellung von invasiver Beatmung auf nicht-invasive Beatmung (NIV) hat für Patienten keine Vorteile und sollte unterbleiben.

? Welche Aussage zur Qualifikation von Pflegediensten und Pflegefachkräften, die beatmete Patienten betreuen, trifft nicht zu?

$\bigcirc$ Pflegefachkräfte sollen Qualifizierungsmaßnahmen mit genau definiertem Inhalt und Umfang absolvieren.

$\bigcirc$ Pflegedienste, die beatmete Patienten betreuen, sollen für jeweils 12 Patienten mindestens eine Fachbereichsleitung mit Zusatzqualifikationen bereithalten.

$\bigcirc$ Pflegehelfer, Arzthelfer oder Heilerziehungspflegende dürfen nicht eigenverantwortlich an der Betreuung beatmeter Patienten mitwirken.

$\bigcirc$ Pflegehelfer, Arzthelfer oder Heilerziehungspflegende sollen vor Übernahme von Grundpflegetätigkeiten spezifisch geschult werden.

$\bigcirc$ Beatmungspflege beinhaltet auch Grundpflege und muss nicht gesondert verordnet werden.

? Welche Aussage zur außerklinischen Beatmung trifft zu?

$\bigcirc$ Die Messung der Sauerstoffsättigung ist in jedem Falle ausreichend.
Eine in der Klinik eingeleitete nicht-invasive Heimbeatmungstherapie aufgrund der Exazerbation einer COPD sollte immer als Heimbeatmungstherapie fortgesetzt werden.

- Nach einer hospitalisationspflichtigen Exazerbation einer COPD sollte bei Betroffenen nach mindestens zwei Wochen nach Krankenhausentlassung nochmals eine Blutgasanalyse erfolgen.

Eine 24-Stunden-Betreuung für invasiv beatmete Patienten in der häuslichen Umgebung sollte aus Kostengründen unterbleiben.

$\bigcirc$ Die Organisation des Überleitmanagements aus der Klinik ist Aufgabe des Hausarztes.

? Welche Aussage zur Versorgung von Patienten mit Störungen des respiratorischen Systems ist zutreffend?

$\bigcirc$ Eine Über- und Fehlversorgung im Bereich außerklinische Beatmung ist praktisch nicht vorhanden, da in Deutschland exzellente Regelungen existieren.

In Deutschland ist eine flächendeckende konzeptionelle und geregelte interdisziplinäre und vor allem intersektorale Versorgung von Patienten mit außerklinischer Beatmung gesichert.

Aufgrund der umfassenden fachärztlichen Betreuung von Patienten mit außerklinischer Beatmung ist der Hausarzt nur gelegentlich eingebunden.

$\bigcirc$ Die Einbindung eines Beatmungszentrums ist bei etablierter außerklinischer Beatmung nicht notwendig.

- Im Sinn einer vorausschauenden Therapieplanung kann das Verfassen einer Patientenverfügung hilfreich sein.

\section{Hier steht eine Anzeige.}

\author{
Springer
}


FORTBILDUNG

\section{Literatur}

1. Lloyd-Owen SJ, Donaldson GC, Ambrosino N et al. Patterns of home mechanical ventilation use in Europe: results from the Eurovent survey. Eur Respir J. 2005;25:1025-31

2. Köhler D. Explosive Zunahme der häuslichen Krankenpflege be Beatmeten und Tracheotomierten. Dtsch Med Wochenschr. 2019;144:1-4

3. Windisch W, J Callegari, C Karagiannidis. Ausserklinische Beatmung in Deutschland. Dtsch Med Wochenschr. 2019;144:743-7

4. Rousseau S. Positionspapier zur aufwendigen ambulanten Versorgung tracheotomierter Patienten mit und ohne Beatmung nach Langzeit-Intensivtherapie. Pneumologie. 2017;71:204-6

5. Karagiannidis C, Strassmann S, Callegari J et al. Epidemiologische Entwicklung der außerklinischen Beatmung: Eine rasant zunehmende Herausforderung für die ambulante und stationäre Patientenversorgung. Dtsch Med Wochenschr. 2019, https://doi.org/10.1055/a-0758-4512

6. Huttmann SE, Magnet FS, Karagiannidis C et al. Quality of life and life satisfaction are severely impaired in patients with longterm invasive ventilation following ICU treatment and unsuccessful weaning. Ann Intensive Care, 2018;8:38

7. Windisch W, Dreher M, Geiseler J et al. S2k-Leitlinie: Nichtinvasive und invasive Beatmung als Therapie der chronischen respiratorischen Insuffizienz - Revision 2017. Pneumologie. 2017:71:722-5

8. WeanNet Study Group. WeanNet: Das Netzwerk von WeaningEinheiten der Deutschen Gesellschaft für Pneumologie und Beatmungsmedizin (DGP). Dtsch Med Wochenschr. 2016:141:e166-e172

9. Schönhofer B, Geiseler J, Dellweg D et al. S2k-Leitlinie prolongiertes Weaning. Pneumologie. 2019;73:723-814

10. Köhnlein T, Windisch W, Köhler D et al. Non-invasive positive pressure ventilation for the treatment of severe stable chronic obstructive pulmonary disease: a prospective, multicentre, randomised, controlled clinical trial. Lancet Respir Med. 2014;2:698-705

11. Murphy PB, Rehal S, Arbane $G$ et al. Effect of home noninvasive ventilation with oxygen therapy vs. oxygen therapy alone on hospital readmission or death after an acute COPD exacerbation. A randomized clinical trial. JAMA. 2017:317:2177-86 